\title{
Physical Comorbidities are Independently Associated with Higher Rates of Psychiatric Readmission in a Chinese Han Population
}

This article was published in the following Dove Press journal: Neuropsychiatric Disease and Treatment

\author{
Chunyu Yang ${ }^{1-4, *}$ \\ Xiaomei Zhong ${ }^{2,4, *}$ \\ Huarong Zhou ${ }^{2,4}$ \\ Zhangying $\mathrm{Wu}^{2,4}$ \\ Min Zhang ${ }^{2,4}$ \\ Yuping Ning ${ }^{1,2,4}$
}

'The First School of Clinical Medicine, Southern Medical University, Guangzhou, Guangdong, People's Republic of China;

${ }^{2}$ The Affiliated Brain Hospital of Guangzhou Medical University, Guangzhou, Guangdong, People's Republic of China; ${ }^{3}$ The Third People's Hospital of Zhongshan, Zhongshan,

Guangdong, People's Republic of China; ${ }^{4}$ Guangdong Engineering Technology Research Center for Translational Medicine of Mental Disorders, Guangzhou, Guangdong, People's Republic of China

*These authors contributed equally to this work

\begin{abstract}
Background: In people with psychosis, physical comorbidities are highly widespread and leading contributors to the untimely death encountered. Readmission rates in psychiatric patients are very high. Somatic comorbidities could be one of the considerable risk factors for psychiatric rehospitalization. Nevertheless, much less is known about the relation between physical comorbidities and psychiatric readmission. We aimed to investigate the association between physical comorbidities and psychiatric readmission in Han Chinese patients with psychiatric disorders.
\end{abstract}

Methods: We used administrative data for January 1, 2009 to December 31, 2018 from the headquarters of the Affiliated Brain Hospital of Guangzhou Medical University to identify adults with schizophrenia, unipolar depression or bipolar disorder discharged from hospital. Data were extracted on sociodemographic and clinical characteristics. The Charlson comorbidity index (CCI) was used to assess the existence of significant physical comorbidity. Cox proportional hazards regression estimated rehospitalization risk after discharge.

Results: A total of 15,620 individuals were included in this study, with the mean age of 35.1 years $(\mathrm{SD}=12.8)$, and readmission occurred for $23.6 \%$ of participants. Survival analysis showed that physical comorbidities were statistically and significantly associated with psychiatric readmission, even after the adjustment for the number of psychiatric comorbidities, other sociodemographic and clinical variables.

Conclusion: Our results suggest that somatic comorbidities are related with higher rates of psychiatric readmission. Hence, to treat psychosis more effectively and to reduce rehospitalization, it is crucial to treat physical comorbidities promptly and adequately. It is absolutely necessary to bring somatic comorbidities to the forefront of psychiatric treatment and research.

Keywords: psychiatric disorder, physical comorbidity, readmission, survival analysis

\section{Introduction}

About half of the psychiatric patients suffered numerous relapses, which has been proved to be related with social function impairment and cognitive deficit. ${ }^{1-3}$ It is worth noting that rehospitalization is an indicator of symptoms relapse. ${ }^{4}$ Risk of rehospitalization is quite high for people with a severe mental illness. ${ }^{5}$ Higher readmission rates can have a substantive effect on mental health spending. ${ }^{6}$ A growing body of evidence indicates that people with mental disorders experience greater physical morbidity, particularly cardiometabolic disease, than the general population. ${ }^{7,8}$ Comorbidity between mental and medical disorders is the rule, not
Correspondence: Yuping Ning The Affiliated Brain Hospital of Guangzhou Medical University, Mingxin Road \#36, Liwan District, Guangzhou, Guangdong, People's Republic of China

Tel $+8620-8126-8720$

Fax $+8620-8189-1391$

Email ningjeny@I26.com 
the exception. ${ }^{9}$ Compared to the general population, the life expectancy of psychiatric patients is reduced -by 15.9 years for males and 12.0 years for females, and $77.7 \%$ of excess deaths are ascribed to physical health conditions. ${ }^{10}$ The comorbidity also has been associated with a poorer functional status, worse quality of life, and a greater healthcare costs. ${ }^{11}$ In addition, it was reported that psychiatric patients were at an increased risk of morbidity due to physical disorders. ${ }^{12}$

Readmission is a commonly used metric of quality of inpatient psychiatric care for policy-makers, government funding agencies, and hospitals deciding on clinical priorities. $^{13}$ Psychiatric reasons for rehospitalization include diagnoses of schizophrenia or bipolar disorder, ${ }^{14}$ aggressiveness or agitation behavior, ${ }^{15}$ harm to self or others, ${ }^{16}$ worsening of psychiatric symptoms, and comorbid psychiatric disorder ${ }^{17}$ and so on. Research has shown that the timeliness of post-discharge follow-up, access to care, the supply of hospital beds, social support, functional status, and substance abuse are associated with psychiatric rehospitalization. ${ }^{18}$ Other studies have indicated that the number of previous psychiatric hospitalizations, ${ }^{19}$ nonadherence to or discontinuance of medication treatment ${ }^{20}$ and duration of hospital stays ${ }^{21}$ are the predictors of risk of rehospitalization to inpatient psychiatric treatment. Comorbidity is one of the most important challenges to modern-day psychiatry and medicine. ${ }^{22}$ There is growing interest among researchers and practitioners in the influence of comorbidity on health-care utilization. ${ }^{23}$ Whereas, the relationship between somatic comorbidity and psychiatric rehospitalization is still poorly understood phenomenon. ${ }^{24}$ Some studies have explored the association between them, but with inconsistent results. For example, Filipcic et al found that chronic somatic comorbidities were linked with higher rates of psychiatric readmission. ${ }^{25}$ Nevertheless, Clements et al reported that there was no association between them. ${ }^{26}$

To the best of our knowledge, predictive factors of psychiatric rehospitalization among patients with mental illness and cooccurring physical disorder are complex and not yet well known in Chinese populations. We have not found conclusive proof that somatic comorbidity affect psychiatric readmission. Currently, the most common approach still separates the physical and mental health care in the field of access to primary preventive interventions, the quality of examination, and adequate treatment. $^{27}$ We hypothesized that physical comorbidity could be a momentous risk factor associated with rehospitalization for people with psychiatric disorders, further research is needed. The present study aimed to address whether physical disorders were associated with higher rates of psychiatric readmission independently of psychiatric comorbidities, other sociodemographic and clinical parameters.

\section{Methods}

\section{Data Sources}

The datasets used for this observational study were obtained from the electronic administrative data from the headquarters of the Affiliated Brain Hospital of Guangzhou Medical University, the oldest Chinese psychiatric hospital, established in 1898.

The research project was conducted in accordance with the Declaration of Helsinki and approved by the ethics committee of the Affiliated Brain Hospital of Guangzhou Medical University (approval number: 2,019,025). The study used retrospective data. The ethics committee of the Affiliated Brain Hospital of Guangzhou Medical University thought that this study met the following conditions for exemption from informed consent: 1 . The purpose of the study was important. 2 . The risk of the study to the subjects was not greater than the minimum risk. 3 . Waiving informed consent would not adversely affect the rights and health of the subjects. 4. The subjects' privacy and personal identity information were protected. 5. If informed consent was required, the study would not be carried out. 6. Whenever possible, the researchers would provide appropriate information to the subjects at an appropriate time after the study. 7. If after consulting the patients' medical records, the researchers found that the patients and their guardians had explicitly refused to use their medical records in future studies, the patients' medical records would not be used.

\section{Study Sample}

The sample included 15,620 Han Chinese patients, who had once or more discharges with a primary diagnosis of schizophrenia (ICD-10 diagnosis of F20) or unipolar depression (ICD-10 F32-F33) or bipolar disorder (ICD-10 F30-F31) between January 1, 2009 and December 31, 2018. The subjects could have psychiatric comorbidities or not. Psychiatric comorbidities included mental and behavioral disorders due to psychoactive substance use (ICD-10 F10-F19), neurotic, stress-related, and somatoform disorders (ICD-10 F40-F48), behavioral syndromes associated with psychological 
disturbances and physical factors (ICD-10 F50-F59), disorders of adult personality and behavior (ICD-10 F60-F69), mental retardation (ICD-10 F70-F71), disorders of psychological development (ICD-10 F80-F89), behavioral and emotional disorders with onset usually occurring in childhood and adolescence (F90-F98). Patients aged between 18 and 65 years during the beginning of the index episode were eligible for the study. We excluded patients who had no primary diagnosis of schizophrenia or unipolar depression or bipolar disorder. Patients who died during their index hospitalization were excluded because they were not any longer at risk of readmission. Furthermore, we excluded the readmissions that happened less than 24 hours after discharge from the index hospitalization to obviate including transfers between medical services within the hospital that could have been recorded as separate hospitalization events in the database. We also excluded those whose hospitalization duration was greater than 5 years as they would have had a shortened follow-up period.

\section{Measures}

Information on sociodemographic characteristics included gender, age, marital status, education and medical settlement mode. Data on clinical characteristics included the length of inpatient stay of the index episode at the Affiliated Brain Hospital of Guangzhou Medical University, the number of previous hospitalizations at the Affiliated Brain Hospital of Guangzhou Medical University, Charlson comorbidity index score, ${ }^{28}$ the number of psychiatric comorbidities and the diagnoses based on ICD-10 codes. For patients who had over more than one discharge with a primary diagnosis of schizophrenia or unipolar depression or bipolar disorder during the period January 1, 2009 to December 31, 2018, we used info on characteristics from the first discharge (except age). We selected an individual's first discharge during the research period as the index admission. We followed the individuals from index date until the first rehospitalization, or December 31, 2018, whichever came first. We only analyzed the first rehospitalization. We did not limit the time for the first admission during the index date.

We used the Charlson comorbidity index (CCI) to assess the presence of significant general physical comorbidity. The Charlson comorbidity measure is one of the most frequently used method in the comparative studies on comorbidity, significantly associating with higher risk of readmission and reflecting the morbidity burden. ${ }^{29,30}$ The $\mathrm{CCI}$ is calculated by assigning a value of $1-6$, for each different comorbidity present (with a higher score signifying greater severity). The total CCI score represents the summation of all individual comorbidity scores. ${ }^{31}$ The ICD 10 codes outlined by Quan et al were used to determine the presence or absence of each comorbidity. ${ }^{32}$

\section{Statistical Analyses}

Data analysis proceeded in two steps as follows:

In step 1, we compared the demographic and clinical features of patients who had at least one readmission during the 10-year period after the index discharge with those who had none, using the Wilcoxon rank tests for continuous variables and chi-square test for categorical variables.

In step 2, we used Cox proportional hazards regression to estimate risks of readmission. CCI score and the number of psychiatric comorbidities were included as categorical variables.

Percentages (\%) means \pm standard deviations (SD) or medians (quartile range) were used to describe the data. We set the level of statistical significance at $\mathrm{P}<0.05$, and all confidence intervals were given at 95\% level. In all cases, we used two-tailed tests. Statistical data analysis was done by SPSS 23.0 statistical software (SPSS Inc., Chicago, United States).

\section{Results}

Sociodemographic characteristics of the 15,620 patients are presented in Table 1. The mean age at the beginning of the index episode was 35.1years $(\mathrm{SD}=12.8)$, ranging from 18 to 65 years. $51.8 \%(n=8097)$ of the sample were male. $68.9 \%$ had less than 12 years of education; $40.7 \%$ were married, while $53.0 \%$ were single and $6.2 \%$ were divorced or widowed. $40.2 \%$ had local or non-local medical insurance. Education history was missing for 1114 (7.1\%) individuals, marital status was missing for $15(0.1 \%)$, and medical settlement mode was only missing for 1 individual.

Table 2 shows 46.0\% $(n=7186)$ had a diagnosis of schizophrenia, $16.4 \%(\mathrm{n}=2566)$ depression, and $37.6 \%$ $(n=5868)$ bipolar disorder. The median length of inpatient treatment was 36 days, with $23.9 \%(n=3740)$ hospitalized for 20 or fewer days, and $31.5 \%(n=4927)$ for $21-40$ days, and $44.6 \%(n=6953)$ for more than 40 days. For the majority of patients $(77.7 \%, n=12,143)$, the index episode was their first admission at the Affiliated Brain Hospital of Guangzhou Medical University, while 20.8\% ( $n=3251)$ had one to two previous admissions, and only $1.4 \%$ $(n=226)$ had three or more. Few of these patients were 
Table I Sociodemographic Characteristics of Patients Discharged Between January I, 2009 and December 3I, 20I8 (n = I5,620)

\begin{tabular}{|c|c|c|c|c|c|}
\hline \multirow[t]{2}{*}{ Variables } & Total & $\begin{array}{l}\text { No Readmission Within } \\
\text { I0 Years }\end{array}$ & $\begin{array}{l}\text { Readmission Within } \\
\text { I0 Years }\end{array}$ & \multirow[t]{2}{*}{$\begin{array}{l}\text { Statistics } \\
\left(\mathbf{X}^{2} / Z\right)\end{array}$} & \multirow[t]{2}{*}{ P-value } \\
\hline & $(n=15,620,100 \%)$ & $(n=\mid I, 933,76.4 \%)$ & $(n=3,687,23.6 \%)$ & & \\
\hline Gender & & & & 10.333 & 0.001 \\
\hline Male & $8,097(51.8 \%)$ & $6,27 \mathrm{I}(52.6 \%)$ & I,826 (49.5\%) & & \\
\hline Female & $7,523(48.2 \%)$ & $5,662(47.4 \%)$ & $\mathrm{I}, 86 \mathrm{I}(50.5 \%)$ & & \\
\hline Age(years) & & & & -3.428 & 0.001 \\
\hline $18-30$ & $7,103(45.5 \%)$ & $5,504(46.1 \%)$ & I,599 (43.4\%) & & \\
\hline $31-45$ & $4,883(31.3 \%)$ & 3,775 (31.6\%) & I,I08 (30.I\%) & & \\
\hline $46-65$ & $3,634(23.3 \%)$ & 2,654 (22.2\%) & $980(26.6 \%)$ & & \\
\hline Mean(SD) & $35.1(12.8)$ & $34.9(12.7)$ & $35.9(13.2)$ & & \\
\hline Median & 32 & 32 & 33 & & \\
\hline Marital status & & & & 4.019 & 0.259 \\
\hline Single & $8,282(53.0 \%)$ & 6,343 (53.2\%) & I,939 (52.6\%) & & \\
\hline Married & $6,360(40.7 \%)$ & $4,864(40.8)$ & I,496 (40.6\%) & & \\
\hline Divorced & 823 (5.3\%) & $605(5.1 \%)$ & $218(5.9 \%)$ & & \\
\hline Widowed & 140 (0.9\%) & $106(0.9 \%)$ & $34(0.9 \%)$ & & \\
\hline Education & & & & 38.650 & $<0.001$ \\
\hline Illiteracy & $107(0.7 \%)$ & $86(0.7 \%)$ & $21(0.6 \%)$ & & \\
\hline Primary & I,237(7.9\%) & $975(8.2 \%)$ & $262(7.1 \%)$ & & \\
\hline Middle school & $4,978(31.9 \%)$ & $3,8 \mid 2(31.9 \%)$ & $1,166(31.6 \%)$ & & \\
\hline High school & $4,438(28.4 \%)$ & $3,197(26.8 \%)$ & $\mathrm{I}, 24 \mathrm{I}(33.7 \%)$ & & \\
\hline University & $3,746(24 \%)$ & $2,804(23.5 \%)$ & $942(25.5 \%)$ & & \\
\hline Medical settlement mode & & & & 276.021 & $<0.001$ \\
\hline Own expense & $9,336(59.8 \%)$ & $7,404(62.0 \%)$ & $1,932(52.4 \%)$ & & \\
\hline Local medical insurance & $5,45 \mathrm{I}(34.9 \%)$ & $3,78 \mathrm{I}(31.7 \%)$ & $\mathrm{I}, 670(45.3 \%)$ & & \\
\hline Non-local medical insurance & $832(5.3 \%)$ & $747(6.3 \%)$ & $85(2.3 \%)$ & & \\
\hline
\end{tabular}

possible to have been hospitalized elsewhere, because greater than $70 \%$ of psychiatric inpatient beds in Guangzhou are at the Affiliated Brain Hospital of Guangzhou Medical University. Altogether, 65.6\% $(n=10,240)$ of patients' CCI score were zero, $29.8 \%$ $(n=4654)$ were one to two, only $4.6 \%(n=726)$ were three or more. The most prevalent physical comorbidities were hepatitis B (8.1\%), hypertensive disease (4.6\%) and diabetes mellitus $(4.2 \%)$. In addition, 93.6\% $(n=14,625)$ of the sample had no psychiatric comorbidities, $6.4 \%$ $(n=995)$ had one or more. The most prevalent psychiatric comorbidities were neurotic, stress-related, and somatoform disorders $(2.6 \%)$, and mental and behavioral disorders due to psychoactive substance use $(2.3 \%)$.

Overall, 23.6\% $(n=3687)$ of the sample suffered from readmission at the Affiliated Brain Hospital of Guangzhou Medical University during the 10-year following the index discharge: $65.6 \%$ had one readmission, $19.8 \%$ had two, $7.2 \%$ had three, and 7.4\% had four or more. Furthermore, $16.0 \%$ $(\mathrm{N}=589)$ of these patients were readmitted within 30 days of discharge from their hospitalization, and 53.4\% ( $\mathrm{N}=1969)$ within one year. Median length of time until rehospitalization for those who were readmitted was 329 days.

Table 3 shows the results of Cox proportional hazards regression. There was no significant difference in length of hospitalization. Variable associated with reduced risk of rehospitalization was older age. The following predictors were associated with an increased risk of readmission: female gender, high school education, having medical insurance, bipolar disorder, more previous hospitalization at the Affiliated Brain Hospital of Guangzhou Medical University and increased CCI. Patients with one CCI score were 1.124 times more likely to be readmitted than those with no CCI score, those with two CCI score were 1.548 times more likely, and those with three or more CCI score were 1.645 times more likely (Figure 1). 
Table 2 Clinical Characteristics of Patients Discharged Between January I, 2009 and December 3I, 2018 (n= I5,620)

\begin{tabular}{|c|c|c|c|c|c|}
\hline \multirow[t]{2}{*}{ Variables } & Total & $\begin{array}{l}\text { No Readmission Within } \\
10 \text { Years }\end{array}$ & $\begin{array}{l}\text { Readmission Within } \\
10 \text { Years }\end{array}$ & \multirow[t]{2}{*}{$\begin{array}{l}\text { Statistics } \\
\left(X^{2} / Z\right)\end{array}$} & \multirow[t]{2}{*}{ P-value } \\
\hline & $\begin{array}{l}(n=15,620 \\
100 \%)\end{array}$ & $(n=11,933,76.4 \%)$ & $(n=3,687,23.6 \%)$ & & \\
\hline Length of stay(days) & & & & -8.359 & $<0.001$ \\
\hline$\leq 20$ & $3,740(23.9 \%)$ & 2,971 (24.9\%) & 769 (20.9\%) & & \\
\hline $21-40$ & 4,927 (31.5\%) & $3,834(32.1 \%)$ & I,093 (29.6\%) & & \\
\hline $4 I-60$ & $2,882(18.5 \%)$ & $2,193(18.4 \%)$ & $689(18.7 \%)$ & & \\
\hline $61-80$ & $1,546(9.9 \%)$ & $\mathrm{I}, \mathrm{I} 57(9.7 \%)$ & $389(10.6 \%)$ & & \\
\hline $81-100$ & I,039 (6.7\%) & $740(6.2 \%)$ & $299(8.1 \%)$ & & \\
\hline$\geq|0|$ & I,486 (9.5\%) & $\mathrm{I}, 038(8.7 \%)$ & 448 (12.2\%) & & \\
\hline Mean(SD) & $58.2(111.7)$ & $57.8(119.2)$ & $59.5(82.7)$ & & \\
\hline Median & 36 & 35 & 40 & & \\
\hline Number of previous admissions & & & & -21.586 & $<0.001$ \\
\hline 0 & $12, \mid 43(77.7 \%)$ & 9,749 (8I.7\%) & $2,394(64.9 \%)$ & & \\
\hline I & $2,944(18.8 \%)$ & I,875 (I5.7\%) & $1,069(29.0 \%)$ & & \\
\hline 2 & $307(2.0 \%)$ & $188(1.6 \%)$ & $119(3.2 \%)$ & & \\
\hline$\geq 3$ & $226(1.4 \%)$ & $121(1.0 \%)$ & $105(2.8 \%)$ & & \\
\hline Charlson comorbidity index score & & & & 41.853 & $<0.001$ \\
\hline 0 & $10,240(65.6 \%)$ & 7,908 (66.3\%) & $2,332(63.2 \%)$ & & \\
\hline I & $3,546(22.7 \%)$ & $2,732(22.9 \%)$ & $814(22.1 \%)$ & & \\
\hline 2 & I, I08 (7.1\%) & $793(6.6 \%)$ & $315(8.5 \%)$ & & \\
\hline$\geq 3$ & $726(4.6 \%)$ & $500(4.2 \%)$ & $226(6.1 \%)$ & & \\
\hline Number of psychiatric comorbidities & & & & 0.102 & 0.750 \\
\hline 0 & $14,625(93.6 \%)$ & II,I77(93.7\%) & $3,448(93.5 \%)$ & & \\
\hline$\geq 1$ & $955(6.4 \%)$ & $756(6.3 \%)$ & $239(6.5 \%)$ & & \\
\hline Diagnosis & & & & 10.824 & 0.004 \\
\hline Schizophrenia & $7,186(46.0 \%)$ & $5,544(46.5 \%)$ & $\mathrm{I}, 642(44.5 \%)$ & & \\
\hline Depression & $2,566(16.4 \%)$ & $1,990(16.7 \%)$ & $576(\mid 5.6 \%)$ & & \\
\hline Bipolar disorder & $5,868(37.6 \%)$ & $4,399(36.9 \%)$ & $1,469(39.8 \%)$ & & \\
\hline Days from discharge to readmission & & & & & \\
\hline$\leq 30$ & N/A & N/A & 589 (16.0\%) & & \\
\hline $31-90$ & $\mathrm{~N} / \mathrm{A}$ & N/A & 321 (8.7\%) & & \\
\hline $91-180$ & N/A & $\mathrm{N} / \mathrm{A}$ & $403(10.9 \%)$ & & \\
\hline$|8|-365$ & $\mathrm{~N} / \mathrm{A}$ & $\mathrm{N} / \mathrm{A}$ & $656(17.8 \%)$ & & \\
\hline $366-730$ & $\mathrm{~N} / \mathrm{A}$ & N/A & 755 (20.5\%) & & \\
\hline$\geq 731$ & N/A & N/A & $963(26.1 \%)$ & & \\
\hline Median & & & 329 & & \\
\hline
\end{tabular}

Abbreviation: N/A, not applicable.

\section{Discussion}

Our study has shown a significant and clinically relevant relationship between physical comorbidities and psychiatric rehospitalization in patients diagnosed with schizophrenia, unipolar depression and bipolar disorder, independent of psychiatric comorbidities, other concerned sociodemographic and clinical parameters in a Chinese Han populations.
Our finding was consistent with other studies that reported the association between general Physical comorbidity and increased risk of psychiatric readmission. ${ }^{21,25,27}$ Discharged patients with severe mental illness diagnoses, a higher CCI score and medical health problems were more probable to experience a subsequent hospital admission. ${ }^{24}$ Some previous studies' outcomes also supported the hypothesis that patients with psychiatric disorders were at increased risk of 
Table 3 Cox Proportional Hazards Analysis of Risk of Readmission

\begin{tabular}{|c|c|c|c|}
\hline Variables & $\begin{array}{l}\text { Hazard } \\
\text { Ratio }\end{array}$ & $95 \% \mathrm{Cl}$ & P-value \\
\hline Age (reference:18-30) & 0.989 & $0.985-0.993$ & $<0.001$ \\
\hline Gender (reference: Male) & 1.129 & $1.057-1.206$ & $<0.001$ \\
\hline \multicolumn{4}{|l|}{ Education (reference: University) } \\
\hline Illiteracy & 0.749 & $0.478-1.174$ & 0.208 \\
\hline Primary & 0.905 & $0.784-1.045$ & 0.174 \\
\hline Middle school & 0.970 & $0.888-1.060$ & 0.503 \\
\hline High school & 1.128 & $1.035-1.230$ & 0.006 \\
\hline \multirow{2}{*}{\multicolumn{4}{|c|}{$\begin{array}{l}\text { Medical settlement mode (reference: } \\
\text { Own expense) }\end{array}$}} \\
\hline & & & \\
\hline Local medical insurance & 1.710 & $1.588-1.842$ & $<0.001$ \\
\hline Non-local medical insurance & 1.247 & $1.001-1.553$ & 0.049 \\
\hline \multicolumn{4}{|l|}{ Diagnosis (reference: Schizophrenia) } \\
\hline Depression & 0.975 & $0.862-1.103$ & 0.693 \\
\hline Bipolar disorder & 1.148 & $1.067-1.235$ & $<0.001$ \\
\hline Length of stay (reference: $\leq 20$ ) & 1.000 & $1.000-1.000$ & 0.704 \\
\hline $\begin{array}{l}\text { Number of previous admissions } \\
\text { (reference:0) }\end{array}$ & 1.279 & $|.247-1.31|$ & $<0.001$ \\
\hline \multicolumn{4}{|l|}{$\begin{array}{l}\text { Charlson comorbidity index score } \\
\text { (reference:0) }\end{array}$} \\
\hline 1 & 1.124 & $1.009-1.253$ & 0.034 \\
\hline 2 & 1.548 & $1.321-1.814$ & $<0.001$ \\
\hline$\geq 3$ & 1.645 & $1.360-1.990$ & $<0.001$ \\
\hline
\end{tabular}

rehospitalization if they had co-occurring somatic conditions. ${ }^{33,34}$ Nevertheless, it is worthy of mentioning that our finding was contrary to the results of the research conducted by Innes et al. ${ }^{31}$ They found no significant association between CCI score and psychiatric readmission. Meanwhile, they reported that older age-group $\left(70^{+}\right.$years $)$was significantly linked with an increased risk of readmission, which appeared to contradict our results. Our study suggested that younger patients had a higher risk of readmission, consistent with a previous study. ${ }^{21}$ Several factors may be responsible for these discrepancies, such as the differences in study population and inclusion criteria, different access to psychiatric and physical health care or differences in economic conditions. Additionally, our study showed that $4.6 \%$ of individuals' CCI score were more than three, which were similar to the finding of Vigod et al. ${ }^{16}$ And we found that $34.4 \%$ of patients' CCI score were more than one. The prevalence of reported physical comorbidities was higher than that found in the research of Shen et $\mathrm{al}^{35}$ and Innes et $\mathrm{al}^{31}$ which reported that, $11.4 \%$ and $18.5 \%$ of patients' CCI score were over one. The differences in characteristics of study population or differences in study protocol may be responsible for these discrepancies.

Physical health comorbidities are a main problem for people suffering from psychiatric disorders. ${ }^{36-38}$ Cooccurring physical illnesses may influence the presentation, diagnosis, treatment outcomes and management of mental disorders in many ways. It may be difficult for clinicians to distinguish the adjustment to physical disorder from the presentation of mental illness since some symptoms characteristic of physical illness (eg, fatigue, pain) may also be psychiatric symptoms. It has been shown that significant co-occurring general medical problems may complicate treatment and enhance a person's overall vulnerability. ${ }^{39}$ More physically ill patients may need polypharmacy, which will give rise to a lower tolerability of psychotropic drugs and higher incidences of therapy discontinuation. ${ }^{40}$ Accordingly, serious, chronic physical disorders may cause lower adherence of mentally ill patients. Moreover, the side effects of somatic therapies not only may interfere with psychiatric treatment, but also impact patients' quality of life, cause negative coping strategies and so on, that may increase the risk of worsened psychiatric symptoms. Ultimately, that will likely lead to relapse. Thus it can be seen, co-occurring physical illnesses are related with higher relapse rates of mental disorders, which indicates a higher probability of rehospitalization.

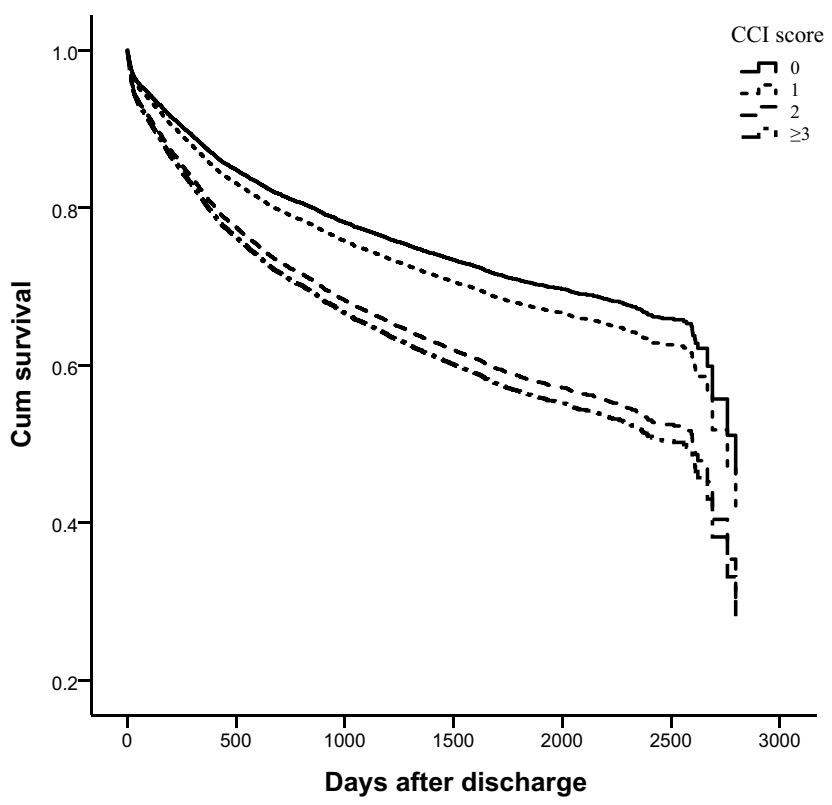

Figure I Survival for days to readmission curves by Charlson comorbidity index $(\mathrm{CCl})$ score. 
Potential reasons for the high physical comorbidity with mental illnesses contain: biological mechanisms, ${ }^{41}$ childhood adversities and the psychiatric disorders themselves increasing the risk for insalubrious lifestyles, ${ }^{42}$ poor sleep quality, ${ }^{43}$ use of medications, which are related with side effects that enhance the risk for somatic diseases, ${ }^{38}$ and poor prevention, detection and treatment of somatic diseases. ${ }^{44}$ The pathways leading to comorbidity of physical and mental disorders are complex and often bidirectional. The course of comorbid physical illnesses and psychiatric disorders could be affected by each other, causing a worsening of either physical illnesses and/or psychiatric disorders, consequently leading to rehospitalization. ${ }^{24}$ As an example, somatic conditions with a high symptom burden, like back pain or migraine, might lead to depression, ${ }^{45}$ while major depressive disorder could stand for a risk factor for developing a somatic condition, such as cardiovascular disease. ${ }^{46}$ Some studies described increased risk of hospital mortality and rehospitalization in patients with comorbid depression and heart condition. ${ }^{47,48}$ Schoepf and Heun reported that people with bipolar disorder had a more serious course of disease and a higher burden of comorbidities. ${ }^{49}$ Individuals living with schizophrenia, bipolar disorder and major depressive disorder displayed a high risk for physical comorbidity, most prevalent diseases comprised diabetes and hypertension, ${ }^{12}$ which were similar to our results. In the present study, we also found that the prevalence of infection with hepatitis B virus (HBV) in subjects was considerably high, which was concordant with previous literature. ${ }^{50-52} \mathrm{HBV}$ is found to increase all-cause mortality by about 2.3 times in the general population. ${ }^{53}$ People with severe mental illness are at higher risk of infection with blood-borne virus, which likely contributes to the excess of mortality observed in this population. ${ }^{52}$ However, infectious diseases have been neglected in psychiatric health research and policy. ${ }^{51}$

In addition, we found that $23.6 \%$ of individuals in our research were readmitted within 10 years. Thereinto, the readmission rate of schizophrenia patients $(22.8 \%)$ was similar to the finding of a large, multicenter, retrospective, observational research in 10 psychiatric hospitals throughout China, which reported that, $18.8 \%$ of patients diagnosed with schizophrenia had at least one rehospitalization during the year after discharge ${ }^{54}$ Furthermore, the result of this analysis was also similar to our previous analysis, ${ }^{19}$ which annual rate of readmission was $12.6 \%$. In both cases, there were no significant differences in length of hospitalization and one of robust predictor of readmission was the number of previous psychiatric hospitalizations. But the readmission rate of our study was substantially lower than that found in the research of Tiihonen et al, ${ }^{55}$ which showed that, over a 20 -year period, $58.8 \%$ of schizophrenia patients were readmitted for psychiatric inpatient care. The readmission rate of unipolar depression patients in our study $(22.4 \%)$ was also lower than another research of Tiihonen et al, ${ }^{56}$ which reported that, over a 16 -year period, $39.7 \%$ of patients diagnosed with unipolar depression had more than one hospitalization. Possible explanations for these generally lower rehospitalization rates in China than the more developed countries include a lower availability of mental hospital beds per person in community, or the cultural norms that more strongly stigmatize mental hospitalization and lead to its avoidance. Additionally, $25.0 \%$ of individuals diagnosed with bipolar disorder in the present study were readmitted. Our analyses indicated that bipolar disorder was associated with an increased risk of rehospitalization, a finding that was similar to a previous research. ${ }^{57}$ Bipolar disorder is a major psychiatric disorder for which the risk of rehospitalization and rate of comorbid physical illnesses are strikingly high. ${ }^{24}$

In China, it is possible that psychiatrists usually pay more attention to psychiatric disorders and are less sensitive to physical fitness. Physical illnesses are seldom analyzed as the possible predictors of mental disorders relapse or rehospitalization. Additionally, stigma towards people who experience mental disorder makes access to health care less easy and quality of careless good. Our research indicates that we should certainly think about the association of physical disorders and optimal treatment outcome in psychiatric patients. Except as modifiable psychotropic medication side effects and lifestyle factors, poorer access to quality of health care remain addressable problems for psychiatric patients. Paying more attentions to these physical illnesses that can worsen mental stability, treatment adherence, and quality of life as well as life expectancy at individual and system level will improve outcomes of these disadvantaged populations. ${ }^{12}$

This study has several limitations that should be mentioned. First of all, in this retrospective, observational study, we derived the CCI score using historical hospitalization data dating back to January 1, 2009. We have not objectively assessed somatic and psychiatric diagnosis. The use of hospital medical records may have limitations in terms of exactitude of the recorded discharge diagnoses, such as data coding errors and incompleteness. Second, 
the readmission rate only addresses rehospitalization at the Affiliated Brain Hospital of Guangzhou Medical University. But most of patients are permanent residents in Guangzhou and the Affiliated Brain Hospital of Guangzhou Medical University accounts for about 70\% of psychiatric beds in Guangzhou. It is the most likely site of readmissions. Third, no detailed clinical information was available regarding treatment received in this study. We also did not collect data on the severity and duration of mental and physical disorders. Additionally, no patients' adherence was analyzed whereas it may be related with a larger number of physical and mental disorders and a higher rates of psychiatric readmission. Future studies should address these problems. Fourth, a previous study has shown that personality disorders may be considered serious mental illnesses and the population prevalence of any personality disorders were common. ${ }^{58}$ Preliminary evidence reveals that personality disorders are associated with increased risk of physical health comorbidities. ${ }^{59}$ Unfortunately, only patients with a primary diagnosis of schizophrenia or unipolar depression or bipolar disorder were included as the targeted population in our study. We have excluded subjects with a primary diagnosis of personality disorder in the present study. A further study of the relationship between physical comorbidities in Han Chinese patients with personality disorders and psychiatric rehospitalization will be needed. Despite the limitations mentioned above, the strength of our study is that it is the first study of the relationship between physical comorbidities and psychiatric readmission in China. It provides some support for mental health policies and clinical practices, and indicates that such a study would be worthwhile.

In summary, our study showed that physical illnesses were associated with higher rates of psychiatric readmission independently of mental comorbidities and other sociodemographic and clinical factors. Clinicians should pay more attention to the treatment of physical disorders in psychiatric patients and need to consider that co-occurring physical illnesses are related with a large range of unfavorable influence, such as the impact on physical health, affecting patients' quality of life and giving rise to recurrence. The integrative, multidisciplinary approach should be necessary for clinical practice. Future studies are needed with more strictly defined and prospective cohort designs which can improve comparability.

\section{Acknowledgments}

We thank Bin Sun for his helpful comments about the statistical analyses. Chunyu Yang and Xiaomei Zhong are co-first authors for this study.

\section{Author Contributions}

All authors made substantial contributions to conception and design, acquisition of data, or analysis and interpretation of data; took part in drafting the article or revising it critically for important intellectual content; agreed to submit to the current journal; gave final approval of the version to be published; and agree to be accountable for all aspects of the work.

\section{Funding}

This study was supported by the following grants: Guangzhou Municipal Psychiatric Disease Clinical Transformation Laboratory (No. 201,805,010,009), Science and Technology Plan Project of Guangdong Province (No. 2019B030316001) and Guangzhou municipal key discipline in medicine (2017-2019). The funding agency had no role in study design, data collection and analysis, decision to publish, or preparation of the manuscript.

\section{Disclosure}

All authors declare no conflicts of interest.

\section{References}

1. Brissos S, Dias VV, Balanza-Martinez V, Carita AI, Figueira ML. Symptomatic remission in schizophrenia patients: relationship with social functioning, quality of life, and neurocognitive performance. Schizophr Res. 2011;129(2-3):133-136. doi:10.1016/j.schres.2011.04.001

2. Barder HE, Sundet K, Rund BR, et al. 10 year course of IQ in first-episode psychosis: relationship between duration of psychosis and long-term intellectual trajectories. Psychiatry Res. 2015;225 (3):515-521. doi:10.1016/j.psychres.2014.11.054

3. Rund BR, Barder HE, Evensen J, et al. Neurocognition and duration of psychosis: a 10-year follow-up of first-episode patients. Schizophr Bull. 2016;42(1):87-95. doi:10.1093/schbul/sbv083

4. Chi MH, Hsiao CY, Chen KC, et al. The readmission rate and medical cost of patients with schizophrenia after first hospitalization - a 10-year follow-up population-based study. Schizophr Res. 2016;170 (1):184-190. doi:10.1016/j.schres.2015.11.025

5. Schennach R, Obermeier M, Meyer S, et al. Predictors of relapse in the year after hospital discharge among patients with schizophrenia. Psychiatr Serv. 2012;63(1):87-90. doi:10.1176/appi.ps.201100084

6. Donisi V, Tedeschi F, Salazzari D, Amaddeo F. Pre- and post-discharge factors influencing early readmission to acute psychiatric wards: implications for quality-of-care indicators in psychiatry. Gen Hosp Psychiatry. 2016;39:53-58. doi:10.1016/j.genhosppsych.2015.10.009

7. Kredentser MS, Martens PJ, Chochinov HM, Prior HJ. Cause and rate of death in people with schizophrenia across the lifespan: a population-based study in Manitoba, Canada. J Clin Psychiatry. 2014;75(2):154-161. doi:10.4088/JCP.13m08711 
8. Gardner-Sood P, Lally J, Smith S, et al. Cardiovascular risk factors and metabolic syndrome in people with established psychotic illnesses: baseline data from the IMPaCT randomized controlled trial. Psychol Med. 2015;45(12):2619-2629. doi:10.1017/S0033291715000562

9. Kessler RC, Berglund P, Chiu WT, et al. The US National Comorbidity Survey Replication (NCS-R): design and field procedures. Int J Methods Psychiatr Res. 2004;13(2):69-92.

10. Lawrence D, Hancock KJ, Kisely S. The gap in life expectancy from preventable physical illness in psychiatric patients in Western Australia: retrospective analysis of population based registers. $B M J$. 2013;346(may211):f2539-f2539. doi:10.1136/bmj.f2539

11. Baumeister H, Hutter N, Bengel J, Harter M. Quality of life in medically ill persons with comorbid mental disorders: a systematic review and meta-analysis. Psychother Psychosom. 2011;80 (5):275-286. doi:10.1159/000323404

12. De Hert M, Correll CU, Bobes J, et al. Physical illness in patients with severe mental disorders. I. Prevalence, impact of medications and disparities in health care. World Psychiatr. 2011;10(1):52-77.

13. Vigod SN, Taylor VH, Fung K, Kurdyak PA. Within-hospital readmission: an indicator of readmission after discharge from psychiatric hospitalization. Can J Psychiatr. 2013;58(8):476-481. doi:10.1177/ 070674371305800806

14. Shafer A. Hospitalization patterns over 30 years across a statewide system of public mental health hospitals: readmission predictors, optimal follow-up period, readmission clusters and individuals with statistically significant high healthcare utilization. Psychiatr $Q$. 2019;90(2):263-273. doi:10.1007/s11126-019-9626-7

15. Rubio-Valera M, Luciano JV, Ortiz JM, Salvador-Carulla L, Gracia A, Serrano-Blanco A. Health service use and costs associated with aggressiveness or agitation and containment in adult psychiatric care: a systematic review of the evidence. BMC Psychiatr. 2015;15:35. doi:10.1186/s12888-015-0417-x

16. Vigod SN, Kurdyak PA, Seitz D, et al. READMIT: a clinical risk index to predict 30-day readmission after discharge from acute psychiatric units. J Psychiatr Res. 2015;61:205-213. doi:10.1016/j.jpsychires.2014.12.003

17. Prince JD, Akincigil A, Kalay E, et al. Psychiatric rehospitalization among elderly persons in the United States. Psychiatr Serv. 2008;59 (9):1038-1045. doi:10.1176/ps.2008.59.9.1038

18. Kansagara D, Englander H, Salanitro A, et al. Risk prediction models for hospital readmission: a systematic review. JAMA. 2011;306 (15):1688-1698. doi:10.1001/jama.2011.1515

19. Zhou Y, Ning Y, Fan N, Mohamed S, Rosenheck RA, He H. Correlates of readmission risk and readmission days in a large psychiatric hospital in Guangzhou, China. Asia Pac Psychiatry. 2014;6 (3):342-349. doi:10.1111/appy.12096

20. Tiihonen J, Mittendorfer-Rutz E, Majak M, et al. Real-world effectiveness of antipsychotic treatments in a nationwide cohort of 29823 patients with schizophrenia. JAMA Psychiatr. 2017;74(7):686-693. doi:10.1001/jamapsychiatry.2017.1322

21. Boaz TL, Becker MA, Andel R, Van Dorn RA, Choi J, Sikirica M. Risk factors for early readmission to acute care for persons with schizophrenia taking antipsychotic medications. Psychiatr Serv. 2013;64(12):1225-1229. doi:10.1176/appi.ps.003382012

22. Sartorius N. Comorbidity of mental and physical disorders: a key problem for medicine in the 21st century. Acta Psychiatr Scand. 2018;137(5):369-370. doi:10.1111/acps. 12888

23. Zulman DM, Asch SM, Martins SB, Kerr EA, Hoffman BB, Goldstein MK. Quality of care for patients with multiple chronic conditions: the role of comorbidity interrelatedness. $J$ Gen Intern Med. 2013;29(3):529-537. doi:10.1007/s11606-013-2616-9

24. Sprah L, Dernovsek MZ, Wahlbeck K, Haaramo P. Psychiatric readmissions and their association with physical comorbidity: a systematic literature review. BMC Psychiatr. 2017;17(1):2. doi:10.1186/s12888-016-1172-3
25. Filipcic I, Simunovic Filipcic I, Ivezic E, et al. Chronic physical illnesses in patients with schizophrenia spectrum disorders are independently associated with higher rates of psychiatric rehospitalization; a cross-sectional study in Croatia. Eur Psychiatry. 2017;43:73-80. doi:10.1016/j.eurpsy.2017.02.484

26. Clements KM, Murphy JM, Eisen SV, Normand SL. Comparison of self-report and clinician-rated measures of psychiatric symptoms and functioning in predicting 1-year hospital readmission. Adm Policy Ment Health. 2006;33(5):568-577. doi:10.1007/s10488-006-0066-y

27. Simunovic Filipcic I, Filipcic I, Glamuzina L, et al. The effect of chronic physical illnesses on psychiatric hospital admission in patients with recurrent major depression. Psychiatry Res. 2019;272:602-608. doi:10.1016/j.psychres.2018.12.178

28. Charlson ME, Charlson RE, Peterson JC, Marinopoulos SS, Briggs WM, Hollenberg JP. The Charlson comorbidity index is adapted to predict costs of chronic disease in primary care patients. J Clin Epidemiol. 2008;61(12):1234-1240. doi:10.1016/j.jclinepi. 2008.01.006

29. Zekry D, Loures Valle BH, Graf C, et al. Prospective comparison of 6 comorbidity indices as predictors of 1-year post-hospital discharge institutionalization, readmission, and mortality in elderly individuals. $J$ Am Med Dir Assoc. 2012;13(3):272-278. doi:10.1016/j.jamda. 2010.11.011

30. Austin SR, Wong YN, Uzzo RG, Beck JR, Egleston BL. Why summary comorbidity measures such as the Charlson comorbidity index and elixhauser score work. Med Care. 2015;53(9):e65-72. doi:10.1097/MLR.0b013e318297429c

31. Innes H, Lewsey J, Smith DJ. Predictors of admission and readmission to hospital for major depression: a community cohort study of 52,990 individuals. J Affect Disord. 2015;183:10-14. doi:10.1016/ j.jad.2015.04.019

32. Quan H, Sundararajan V, Halfon P, Fong A, Burnand B, Luthi J-C. Coding algorithms for defining comorbidities in ICD-9-CM and ICD-10 administrative data. Med Care. 2005;43:1130-1139. doi:10.1097/01.mlr.0000182534.19832.83

33. Chwastiak LA, Davydow DS, McKibbin CL, et al. The effect of serious mental illness on the risk of rehospitalization among patients with diabetes. Psychosomatics. 2014;55(2):134-143. doi:10.1016/j. psym.2013.08.012

34. van Walraven C, Austin PC, Jennings A, Quan H, Forster AJ. A modification of the Elixhauser comorbidity measures into a point system for hospital death using administrative data. Med Care. 2009;47(6):626-633. doi:10.1097/MLR.0b013e31819432e5

35. Shen SP, Chen YC, Wu HC, et al. A comparison of hospital and community stay in patients who underwent compulsory admission before and after the 2007 amendment to the mental health act in Taiwan. Int J Law Psychiatry. 2018;58:87-96. doi:10.1016/j. ijlp.2018.02.012

36. Correll CU, Detraux J, De Lepeleire J, De Hert M. Effects of antipsychotics, antidepressants and mood stabilizers on risk for physical diseases in people with schizophrenia, depression and bipolar disorder. World Psychiatr. 2015;14(2):119-136. doi:10.1002/ wps. 20204

37. Docherty M, Stubbs B, Gaughran F. Strategies to deal with comorbid physical illness in psychosis. Epidemiol Psychiatr Sci. 2016;25 (3):197-204. doi:10.1017/S2045796016000056

38. De Hert M, Cohen D, Bobes J, et al. Physical illness in patients with severe mental disorders. II. Barriers to care, monitoring and treatment guidelines, plus recommendations at the system and individual level. World Psychiatr. 2011;10(2):138-151. doi:10.1002/j.2051-5545. 2011.tb00036.x

39. Druss BG, Mauer BJ. Health care reform and care at the behavioral health-primary care interface. Psychiatr Serv. 2010;61(11):1087-1092. doi:10.1176/ps.2010.61.11.1087 
40. Owen-Smith A, Stewart C, Green C, et al. Adherence to common cardiovascular medications in patients with schizophrenia vs. patients without psychiatric illness. Gen Hosp Psychiatry. 2016;38 (undefined):9-14. doi:10.1016/j.genhosppsych.2015.07.010

41. Dieset I, Andreassen OA, Haukvik UK. Somatic comorbidity in schizophrenia: some possible biological mechanisms across the life span. Schizophr Bull. 2016;42(6):1316-1319. doi:10.1093/schbul/ sbw028

42. Scott KM, Von Korff M, Angermeyer MC, et al. Association of childhood adversities and early-onset mental disorders with adult-onset chronic physical conditions. Arch Gen Psychiatry. 2011;68(8):838-844. doi:10.1001/archgenpsychiatry.2011.77

43. Lederman O, Ward PB, Firth J, et al. Does exercise improve sleep quality in individuals with mental illness? A systematic review and meta-analysis. J Psychiatr Res. 2019;109:96-106. doi:10.1016/j. jpsychires.2018.11.004

44. van Hasselt FM, Oud MJ, Loonen AJ. Improvement of care for the physical health of patients with severe mental illness: a qualitative study assessing the view of patients and families. BMC Health Serv Res. 2013;13(1):426. doi:10.1186/1472-6963-13-426

45. Patten SB. Long-term medical conditions and major depression in a Canadian population study at waves 1 and 2. J Affect Disord. 2001;63(1-3):35-41. doi:10.1016/S0165-0327(00)00186-5

46. Patten SB, Williams JVA, Lavorato DH, Modgill G, Jetté N, Eliasziw M. Major depression as a risk factor for chronic disease incidence: longitudinal analyses in a general population cohort. Gen Hosp Psychiatry. 2008;30(5):407-413. doi:10.1016/j.genhosppsych.2008.05.001

47. Andrés E, García-Campayo J, Magán P, et al. Psychiatric morbidity as a risk factor for hospital readmission for acute myocardial infarction: an 8-year follow-up study in Spain. Int $J$ Psychiatr Med. 2012;44(1):63-75. doi:10.2190/PM.44.1.e

48. Jiang W, Alexander J, Christopher E, et al. Relationship of depression to increased risk of mortality and rehospitalization in patients with congestive heart failure. Arch Intern Med. 2001;161(15):1849-1856. doi:10.1001/archinte.161.15.1849

49. Schoepf D, Heun R. Bipolar disorder and comorbidity: increased prevalence and increased relevance of comorbidity for hospital-based mortality during a 12.5-year observation period in general hospital admissions. J Affect Disord. 2014;169:170-178. doi:10.1016/j.jad.2014.08.025

50. Carmo RA, Melo AP, Dezanet LN, de Oliveira HN, Cournos F, Guimaraes MD. Correlates of hepatitis B among patients with mental illness in Brazil. Gen Hosp Psychiatry. 2014;36(4):398-405. doi:10.1016/j.genhosppsych.2014.03.001
51. Hughes E, Bassi S, Gilbody S, Bland M, Martin F. Prevalence of HIV, hepatitis B, and hepatitis $\mathrm{C}$ in people with severe mental illness: a systematic review and meta-analysis. Lancet Psychiatry. 2016;3 (1):40-48. doi:10.1016/S2215-0366(15)00357-0

52. Bauer-Staeb C, Jorgensen L, Lewis G, Dalman C, Osborn DPJ, Hayes JF. Prevalence and risk factors for HIV, hepatitis B, and hepatitis $\mathrm{C}$ in people with severe mental illness: a total population study of Sweden. Lancet Psychiatry. 2017;4(9):685-693. doi:10.1016/S2215-0366(17)30253-5

53. Duberg A-S, Törner A, Daviðsdóttir L, et al. Cause of death in individuals with chronic $\mathrm{HBV}$ and/or $\mathrm{HCV}$ infection, a nationwide community-based register study. J Viral Hepat. 2008;15(7):538-550. doi:10.1111/j.1365-2893.2008.00982.x

54. Xiao J, Mi W, Li L, Shi Y, Zhang H. High relapse rate and poor medication adherence in the Chinese population with schizophrenia: results from an observational survey in the People's Republic of China. Neuropsychiatr Dis Treat. 2015;11:1161-1167. doi:10.2147/ NDT.S72367

55. Tiihonen J, Taipale H, Mehtala J, Vattulainen P, Correll CU, Tanskanen A. Association of antipsychotic polypharmacy vs monotherapy with psychiatric rehospitalization among adults with schizophrenia. JAMA Psychiatr. 2019;76(5):499-507. doi:10.1001/ jamapsychiatry.2018.4320

56. Tiihonen J, Tanskanen A, Hoti F, et al. Pharmacological treatments and risk of readmission to hospital for unipolar depression in Finland: a nationwide cohort study. Lancet Psychiatry. 2017;4(7):547-553. doi:10.1016/S2215-0366(17)30134-7

57. Becker MA, Boaz TL, Andel R, Hafner S. Risk of early rehospitalization for non-behavioral health conditions among adult medicaid beneficiaries with severe mental illness or substance use disorders. J Behav Health Serv Res. 2017;44(1):113-121. doi:10.1007/s11414016-9516-9

58. Quirk SE, Berk M, Chanen AM, et al. Population prevalence of personality disorder and associations with physical health comorbidities and health care service utilization: a review. Personal Disord. 2016;7(2):136-146. doi:10.1037/per0000148

59. Quirk SE, El-Gabalawy R, Brennan SL, et al. Personality disorders and physical comorbidities in adults from the United States: data from the national epidemiologic survey on alcohol and related conditions. Soc Psychiatry Psychiatr Epidemiol. 2015;50 (5):807-820. doi:10.1007/s00127-014-0974-1
Neuropsychiatric Disease and Treatment

\section{Publish your work in this journal}

Neuropsychiatric Disease and Treatment is an international, peerreviewed journal of clinical therapeutics and pharmacology focusing on concise rapid reporting of clinical or pre-clinical studies on a range of neuropsychiatric and neurological disorders. This journal is indexed on PubMed Central, the 'PsycINFO' database and CAS, and is the official journal of The International Neuropsychiatric Association (INA). The manuscript management system is completely online and includes a very quick and fair peer-review system which is all easy to use. Visit http://www.dovepress.com/testimonials.php to read real quotes from published authors. 